\title{
Free Search in Multidimensional Space II
}

\author{
Kalin Penev ${ }^{(\bowtie)}$ \\ Technology School, Maritime and Technology Faculty, Southampton Solent \\ University, East Park Terrace, Southampton SO14 0YN, UK \\ Kalin.Penev@solent.ac.uk
}

\begin{abstract}
Recent publications suggest that resolving multidimensional tasks where optimisation parameters are hundreds and more faces unusual computational limitation. In the same time optimisation algorithms, which perform well on tasks with low number of dimensions, when are applied to high dimensional tasks require infeasible period of time and computational resources. This article presents a novel investigation on Differential Evolution and Particle Swarm Optimisation with enhanced adaptivity and Free Search applied to 200 dimensional versions of three scalable, global, real-value, numerical tests, which optimal values are dependent on dimensions number and virtually unknown for variety of dimensions. The aim is to: (1) identify computational limitations which numerical methods could face on 200 dimensional tests; (2) identify relations between test complexity and period of time required for tests resolving; (3) discover unknown optimal solutions; (4) identify specific methods' peculiarities which could support the performance on high dimensional tasks. Experimental results are presented and analysed.
\end{abstract}

Keywords: Free Search • Differential Evolution • Particle Swarm Optimization • Multidimensional dimensions optimization

\section{Introduction}

This article presents a novel investigation on two hundred dimensional (200D) versions of three scalable real-value numerical tests. Explored are real coded optimisation algorithms Free Search (FS) [10], Differential Evolution (DE) [15] and Particle Swarm Optimisation (PSO) [3]. It continues the efforts on multidimensional optimisation published earlier [12]. The number of potential solution for 200 dimensions (200D), similarly to one hundred dimensional tests is large. This makes these tasks difficult for identification of the optimal solutions and their clarification with acceptable level of precision.

Substantial research efforts are involved in evaluation and improvement of existing and design of new methods capable of resolving multidimensional tasks [5, 7-9, $12-14,16,17]$.

Publications suggest that assessment of evolutionary methods, is limited to 10,30 , 50 and 100 dimensions [7] and methods which perform well on numerical tests with up to 10,30 and 50 dimensions are suffering insuperable stagnation on 100 dimensional tests [7, 12]. When applied to multidimensional tasks with hundreds of parameters well-known methods face difficulties such as: - need for large number of objective 
function evaluations (OFE); - need for large computational resources; - need for large period of time for calculations; - inability to identify optimal solution; - inability to clarify optimal solution with appropriate level of precision [12]. In summary identification of optimal solutions with acceptable level of precision and within acceptable period of time for more than 100 dimensions seems a great challenge and need additional research efforts.

The aim of this study is also to continue evaluation of DE and PSO with enhanced abilities for adaptation and FS, to avoid stagnation and trapping in local suboptimal solution, to identify minimal number of iterations required to resolve 200 dimensional optimisation tests with acceptable precision. For this purpose three scalable, global, real-value, numerical tests, which optimal values are dependent on dimensions number and virtually unknown for 200 dimensions are used - Schwefel [1], Michalewicz [6] and Norwegian [2] tests.

\section{Test Problems}

Tests selection uses the following criteria:

- must be scalable to 200 dimensions;

- must be for global optimisation with many local suboptimal solutions;

- must not provide initial knowledge for optimal solution value and location;

- optimal solution must be dependent on dimensions number.

The test, which meets the above criteria and selected for this investigation are presented below.

\subsection{Schwefel Test}

This test function referred in the literature [1] is:

$$
f\left(x_{i}\right)=418.9829^{*} n-\sum_{i=1}^{n} x_{i} \sin \left(\sqrt{\left|x_{i}\right|}\right)
$$

where $n$ is the number of dimensions and $-500 \leq x_{i} \leq 500, i=1, \ldots, n$. The maximum is dependent on dimensions number and for $n=200$ is unknown.

\subsection{Michalewicz Test Function}

The Michalewicz test function [6] is global optimisation problem. In this study it is transformed for maximization.

$$
f\left(x_{i}\right)=\sum_{i=1}^{n} \sin \left(x_{i}\right)\left(\sin \left(i x_{i}^{2} / \pi\right)\right)^{2 m}
$$


where search space is defined as $0 \leq x_{i} \leq \pi, \mathrm{i}=1, \ldots, n, m=10$. The maximum is dependent on dimensions number and for $n=200$ is unknown.

\subsection{Norwegian Test Function}

Norwegian test function is global test problem [2].

$$
f\left(x_{i}\right)=\prod_{i=1}^{n}\left(\cos \left(\pi x_{i}^{3}\right)\left(\frac{99+x_{i}}{100}\right)\right)
$$

where search space borders are defined by $-1.1<x_{i}<1.1, \mathrm{i}=1, \ldots, n$. The maximum is dependent on dimensions number and for $n=200$ is unknown.

\section{Optimization Methods}

In this study three optimization methods are used - FS, DE and PSO. In order to clarify the abilities to explore and resolve multidimensional global tasks this section focuses on the event modification of these algorithms.

\subsection{Free Search}

Free Search is adaptive heuristic method [10] for real coded optimisation. It is based on a conceptual model, which is different from other methods. In Free Search optimisation process of continuous search space is organised in sequence of short explorations within continuous neighbouring area.

Modification strategy for FS is generated according to the Eqs. (4) and (5):

$$
x_{t j i}=x_{0 j i}-\Delta \mathrm{x}_{t j i}+2^{*} \Delta \mathrm{x}_{t j i}{ }^{*} \operatorname{random}_{t j i}(0,1)
$$

$x_{0 j i}$ is an initial or previous location marked as good. $\operatorname{random}_{t j i}(0,1)$ is a random value between 0 and $1 . t$ is current step $t=1, \ldots, T, T$ is the step limit per exploration. $\Delta \mathrm{x}_{t j i}$ is the step. The step size generation is:

$$
\Delta \mathrm{x}_{t j i}=R_{j i}{ }^{*}\left(\operatorname{Xmax}_{i}-\operatorname{Xmin}_{i}\right)^{*} \operatorname{random}_{t j i}(0,1)
$$

where $R_{j i}$ is a variable value of the neighbour space radius $R_{j i}=[R \min , R \max ] . X \min _{i}$ and $\operatorname{Xmax}_{i}$ are the search space borders. $\operatorname{random}_{t j i}(0,1)$ is a random value between 0 and 1 . The search space borders restrict the probability for access to any location within the search space, only. Variation of $R_{j i}$ higher than one exceeds the search space borders and guarantees non-zero probability for access to any location within the search space. It guarantees a probabilistic transaction rule for exploration of the whole space. FS is implemented with a population of 10 individuals and the explorations are 5 steps, for all experiments. The sense is random in the highest $10 \%$ of the sensibility, and the neighbouring space varies from 0.5 to 1.5 with step 0.1 [10]. 


\subsection{Differential Evolution}

Differential Evolution could be classified as a simple and powerful real value optimisation method. Explored solutions in DE are called vectors. DE selects from current population target, donor and differential vectors. From these vectors DE generates a new trial vector, which replaces the target vector, if it is better, in the new population. The authors proposed several strategies for generation of a trail vector [15].

In line with the literature target vector is denoted as $X_{\mathrm{k}}$, differential vectors are $X_{\mathrm{i}}$ and $X_{\mathrm{j}}$, and differential factor (weight) is $F$. Every pair of vectors $\left(X_{\mathrm{i}}, X_{\mathrm{j}}\right)$ in the primary array defines a difference $X_{\mathrm{i}}-X_{\mathrm{j}}$. These two vectors are usually chosen randomly, their weighted difference is used to perturb another vector in the primary array, $X_{\mathrm{k}}^{\prime}$ :

$$
X_{\mathrm{k}}^{\prime}=X_{\mathrm{k}}+F\left(X_{\mathrm{i}}-X_{\mathrm{j}}\right)
$$

$F$ scales the difference achieved from $X_{\mathrm{i}}-X_{\mathrm{j}}$. An effective variation of this scheme involves keeping track of the best vector so far noted as $X^{*}$. This can be combined with $X_{\mathrm{k}}$ and then perturbed, producing:

$$
X_{\mathrm{k}}^{\prime}=X_{\mathrm{k}}+F\left(X^{*}-X_{\mathrm{k}}\right)+F\left(X_{\mathrm{i}}-X_{\mathrm{j}}\right) .
$$

Several modification strategies are originally proposed:

$$
\begin{gathered}
X_{\mathrm{k}}^{\prime}=X_{\mathrm{k}}+F\left(X_{\mathrm{i}}-X_{\mathrm{j}}\right), \\
X_{\mathrm{k}}^{\prime}=X^{*}+F\left(X_{\mathrm{i}}-X_{\mathrm{j}}\right), \\
X_{\mathrm{k}}^{\prime}=X_{\mathrm{k}}+F\left(X^{*}-X_{\mathrm{k}}\right)+F\left(X_{\mathrm{i}}-X_{\mathrm{j}}\right), \\
X_{\mathrm{k}}^{\prime}=X^{*}+F\left(X_{\mathrm{i}}-X_{\mathrm{j}}+X_{\mathrm{n}}-X_{\mathrm{m}}\right), \\
X_{\mathrm{k}}^{\prime}=X_{\mathrm{k}}+F\left(X^{*}-X_{\mathrm{k}}+X_{\mathrm{n}}-X_{\mathrm{m}}\right) .
\end{gathered}
$$

DE is implemented with population of 10 individuals and explored with strategy at Eq. (12). All individuals are subject of replacement. The crossover probability is 0.5. Differential factor varies from 0.5 to 1.5 with step 0.1 .

\subsection{Particle Swarm Optimisation}

Particle Swarm Optimisation could be classified as real value optimisation method motivated from simulation of social behaviour of a group of individuals [3]. PSO generates new values for all particles (individuals) in the swarm (population). It memorises the previous individual and social (swarm) experience and it uses them for generation of new particles.

Earlier the modification strategy of PSO has been improved by use of the original concept for the so called inertia parameter that increases the overall performance of 
PSO [3]. With the addition of the inertia factor, $w$, [4] the particles are manipulated according to the following equations:

$$
\begin{gathered}
v_{\mathrm{id}}=w * v_{\mathrm{id}}+n_{1} * \operatorname{random}(0,1) *\left(P_{\mathrm{id}}-x_{\mathrm{id}}\right)+n_{2} * \operatorname{random}(0,1) *\left(g_{\mathrm{d}}-x_{\mathrm{id}}\right) \\
x_{\mathrm{id}}=x_{\mathrm{id}}+v_{\mathrm{id}}
\end{gathered}
$$

Where the constants $n_{1}$ and $n_{2}$ determine the relative influence of the social and cognitive components, and are usually both set the same to give each component equal weight as the cognitive and social learning rate. PSO is implemented and explored with inertia parameter. The inertia parameter varies from 0.5 to 1.5 with step 0.1 . It has a population of 10 individuals for all experiments. The individual and the social learning factors are 2 for all experiments.

\section{Experimental Methodology}

Methodology aims to identify minimal number of OFE required to achieve optimal result with acceptable level of precision. Selected test are evaluated in two series of 320 experiments, with start from different random locations. First series are limited to $2.10^{6}$ and second to $2.10^{8}$ OFE. Achieved experimental results are presented and compared for maximal achieved result.

\section{Experimental Results}

Achieved from two series of 320 experiments results on Schwefel, Michalewicz and Norwegian test functions are presented in Tables 1, 2, 3, and 4 below.

Table 1. Maximal results from 320 experiments

\begin{tabular}{l|l|l|l|l}
\hline & $\begin{array}{l}\text { Function } \\
\text { evaluations }\end{array}$ & FS & DE & PSO \\
\hline \multirow{2}{*}{ Schwefel } & $2.10^{6}$ & 167582 & 146154 & 139643 \\
\cline { 2 - 5 } & $2.10^{8}$ & 167592 & 146372 & 140457 \\
\hline \multirow{2}{*}{ Michalewicz } & $2.10^{6}$ & 199.473 & 158.7 & 153.809 \\
\cline { 2 - 5 } & $2.10^{8}$ & 199.612 & 162.486 & 156.381 \\
\hline \multirow{2}{*}{ Norwegian } & $2.10^{6}$ & 0.553932 & 0.19664 & 0.0209277 \\
\cline { 2 - 5 } & $2.10^{8}$ & 1.00007 & 0.203317 & 0.021299 \\
\hline
\end{tabular}


Table 2. Mean results from 320 experiments

\begin{tabular}{l|l|l|l|l}
\hline & Function evaluations & FS & DE & PSO \\
\hline \multirow{2}{*}{ Schwefel } & $2.10^{6}$ & 167577.8906 & 128025.4094 & 107220.6172 \\
\cline { 2 - 5 } Michalewicz & $2.10^{8}$ & 167591.9969 & 131360.6531 & 108331.9978 \\
\cline { 2 - 5 } & $2.10^{6}$ & 199.3335375 & 81.97717438 & 57.07641063 \\
\hline \multirow{2}{*}{ Norwegian } & $2.10^{8}$ & 199.6084094 & 100.1925019 & 60.7117175 \\
\cline { 2 - 5 } & $2.10^{6}$ & 0.478959472 & 0.042988971 & $9.26984 \mathrm{E}-05$ \\
\hline
\end{tabular}

Table 3. Standard deviation from 320 experiments

\begin{tabular}{l|l|l|l|l}
\hline & Function evaluations & FS & DE & PSO \\
\hline \multirow{2}{*}{ Schwefel } & $2.10^{6}$ & 1.570401894 & 11641.83834 & 16045.21447 \\
\cline { 2 - 5 } Michalewicz & $2.10^{8}$ & 0.967082 & 9190.399551 & 16166.05376 \\
\cline { 2 - 5 } & $2.10^{6}$ & 0.063176775 & 35.83038048 & 40.39638912 \\
\cline { 2 - 5 } Norwegian & $2.10^{8}$ & 0.001784807 & 40.55275988 & 42.02935262 \\
\cline { 2 - 5 } & $2.10^{6}$ & 0.026979583 & 0.072504813 & 0.001185493 \\
\cline { 2 - 5 } & $2.10^{8}$ & 0.009963986 & 0.087516196 & 0.001205768 \\
\hline
\end{tabular}

Table 4. Standard deviation from 320 experiments in $\%$ from maximum

\begin{tabular}{l|l|l|l|l}
\hline & $\begin{array}{l}\text { Function } \\
\text { evaluations }\end{array}$ & FS & DE & PSO \\
\hline \multirow{2}{*}{ Schwefel } & $2.10^{6}$ & $0.0000973 \%$ & $6.9465358 \%$ & $9.5739739 \%$ \\
\cline { 2 - 5 } & $2.10^{8}$ & $0.0000577 \%$ & $5.4837937 \%$ & $9.6460772 \%$ \\
\hline \multirow{2}{*}{ Michalewicz } & $2.10^{6}$ & $0.0031649 \%$ & $17.9500132 \%$ & $20.2374552 \%$ \\
\cline { 2 - 5 } & $2.10^{8}$ & $0.0000894 \%$ & $20.3157925 \%$ & $21.0555240 \%$ \\
\hline & $2.10^{6}$ & $2.6977694 \%$ & $7.24997380 \%$ & $0.1185410 \%$ \\
\cline { 2 - 5 } & $2.10^{8}$ & $0.9963288 \%$ & $8.75100702 \%$ & $0.1205683 \%$ \\
\hline
\end{tabular}

\section{Discussion}

Analysis of experimental results suggests that on Schwefel and Michalewicz test functions DE and PSO stagnate in suboptimal solutions for all experiments limited to $2.10^{6}$ and $2.10^{8}$ OFE. Reasons for this could be a subject of further research. On Schwefel and Michalewicz test FS achieves optimal solution for all experiments limited to $2.10^{6}$ and $2.10^{8}$ OFE. For experiments limited to $2.10^{8}$ OFE FS refines the precision of the results for Michalewicz test with $0.3 \%$ and for Schwefel test with $0.02 \%$.

Solving 200 dimensional Schwefel and Michalewicz tests for each run confirms good exploration abilities of FS. It indirectly suggests that these tasks could be resolved 
within less number of OFE, which could be a subject of further research. For Norwegian test function used implementations of DE and PSO stagnate in suboptimal solutions for all experiments limited to $2.10^{6}$ and $2.10^{8}$ OFE. DE and PSO had also difficulties on 2 dimensional [11] and 100D versions [12] of this test. Reasons for this could be a subject of further research.

In contrast FS confirms its abilities to avoid stagnation and escape from trapping in suboptimal local areas. For the first series of 320 experiments on Norwegian test limited to $2.10^{6}$ OFE FS does not reach optimal solution. However for tests limited to $2.10^{8}$ OFE from 320 runs with different start locations FS reaches 26 times optimal solutions with acceptable precision (above 1.00004). This corresponds to $8 \%$ probability for success. Whether high probability for success will be reached for higher number of OFE could be a subject of further research. Overall this is a good illustration of the effectiveness of FS modification strategy, which guarantees non-zero probability for access to the whole search space during entire optimization process.

Other essential issue is a period of time required for completion of optimization task. For experiments limited to $2.10^{8}$ OFE average periods of time in minutes, from 320 experiments, required for completion of one experiment on Schwefel, Michalewicz and Norwegian test are presented in Table 5 below.

Table 5. Average period of time in minutes for $2.10^{8}$ objective function evaluations

\begin{tabular}{l|l|l|r|l}
\hline & Function evaluations & FS & DE & PSO \\
\hline Schwefel & 200000000 & $31 \mathrm{~min}$ & $82 \mathrm{~min}$ & $221 \mathrm{~min}$ \\
\hline Michalewicz & 200000000 & $84 \mathrm{~min}$ & $131 \mathrm{~min}$ & $230 \mathrm{~min}$ \\
\hline Norwegian & 200000000 & $15 \mathrm{~min}$ & $62 \mathrm{~min}$ & $145 \mathrm{~min}$ \\
\hline
\end{tabular}

Time periods in Table 5 are measured on processor Intel i7 3960x overclocked to 4.5 GHz and memory G. Skill TridentX at $1866 \mathrm{MHz}$, motherboard ASUS Rampage VI and solid state disk - SanDisk Extreme SSD SATA III. Experiments are completed simultaneously in hyper-treading processor mode. The results presented in Table 5 indicate that FS completes the tests faster than DE and PSO. In the same time Table 1 shows that FS reaches optimal solutions and DE and PSO did not.

Achieved results confirm that FS process is more effective than logically and analytically organised search processes on uncertain and unknown global multidimensional problems due to the expiration, during the search process, of the knowledge, on which logical processes are based.

\section{Conclusion}

This article presents experimental evaluation of FS, DE and PSO on hard global multidimensional tests. Identified are minimal numbers of iterations for which selected test could be resolved with certain probability. Achieved results suggest that FS completed the same number of objective function evaluation for less time than DE and PSO and riches optimal solutions with $100 \%$ probability for Schwefel and 
Michalewicz tests and $8 \%$ for Norwegian test, while DE and PSO success is $0 \%$ for all tests. Further investigation could focus on evaluation and measure of time and computational resources sufficient for completion of other multidimensional tasks or for higher number of dimensions until reaching the limits of modern computational systems. Algorithms analysis and improvement could be also subject of future research.

Acknowledgements. I would like to thank to my students Asim Al Nashwan, Dimitrios Kalfas, Georgius Haritonidis, and Michael Borg for the design, implementation and overclocking of desktop PC used for completion of the experiments presented in this article.

\section{References}

1. Bäck, T., Schwefel, H.P.: An overview of evolutionary algorithms for parameter optimization. Evol. Comput. 1(1), 1-23 (1993)

2. Brekke, E.F.: Complex Behaviour in Dynamical Systems, pp. 37-38. The Norwegian University of Science and Technology (2004). http://www.academia.edu/545835/COMPLEX_ BEHAVIOR_IN_DYNAMICAL_SYSTEMS. Accessed 29 May 2014

3. Eberhart, R., Kennedy, J.: Particle swarm optimisation. In: Proceedings of the 1995 IEEE International Conference on Neural Networks, vol. 4, pp. 1942-1948. IEEE Press (1995)

4. Eberhart, R., Shi, Y.: Comparing inertia weights and construction factors in particle swarm optimization. In: Proceedings of the 2000 CEC, pp. 84-89 (2000)

5. Hendtlass, T.: Particle swarm optimization and high dimensional problem spaces, In: IEEE Congress on Evolutionary Computation, CEC 2009, pp. 1988-1994 (2009)

6. Hedar, A.-R.: Test functions for unconstrained global optimization (2014). http://wwwoptima.amp.i.kyoto-u.ac.jp/member/student/hedar/Hedar_files/TestGO_files/Page2376.htm. Accessed 29 May 2014

7. Liang, J.J., Qu, B.-Y., Suganthan, P.N.: Problem definitions and evaluation criteria for the CEC 2014 special session and competition on single objective real-parameter numerical optimization. Technical report 201311, December 2013 (2014). http://www.ntu.edu.sg/ home/EPNSugan/index_files/CEC2014/CEC2014.htm. Accessed 16 September 2014

8. MacNish, C., Yao, X.: Direction matters in high-dimensional optimisation. In: IEEE Congress on Evolutionary Computation, pp. 2372-2379 (2008)

9. Noman, N., Iba, H.: Enhancing differential evolution performance with local search for high dimensional function optimization. In: Proceedings of the 2005 Conference on Genetic and Evolutionary Computation, pp. 967-974 (2005)

10. Penev, K.: Free Search of Real Value or How to Make Computers Think. St. Qu, Southampton (2008). ISBN 978-0-9558948-0-0

11. Penev, K.: Adaptive intelligence - essential aspects. J. Inf. Technol. Control VII(4), 8-17 (2009). ISSN 1312-2622

12. Penev, K.: Free search - comparative analysis 100. Int. J. Metaheuristics 3(1), 22-33 (2013)

13. Liu, P., Lau, F., Lewis, M.J., Wang, C.-1.: A new asynchronous parallel evolutionary algorithm for function optimization. In: Guervós, J.J.M., Adamidis, P.A., Beyer, H.-G., Fernández-Villacañas, J.-L., Schwefel, H.-P. (eds.) PPSN 2002. LNCS, vol. 2439, pp. 401-410. Springer, Heidelberg (2002)

14. Liu, P., Lewis, M.J.: Communication aspects of an asynchronous parallel evolutionary algorithm. In: Proceedings of the Third International Conference on Communications in Computing, Las Vegas, NV, 24-27 June 2002, pp. 190-195 (2002) 